\title{
Rutin inhibits coronary heart disease through ERK1/2 and Akt signaling in a porcine model
}

\author{
LIN LV $^{1 *}$, YUCAI YAO ${ }^{2 *}$, GANG ZHAO ${ }^{3}$ and GUIYUE ZHU ${ }^{3}$ \\ ${ }^{1}$ The First Clinical Institute, Jining Medical University, Jining, Shandong 272067; ${ }^{2}$ Department of Cardiology, \\ Shandong Provincial Qianfoshan Hospital, Shandong University, Jinan, Shandong 250014; ${ }^{3}$ Department of Cardiology, \\ Shandong Provincial Hospital Affiliated to Shandong University, Jinan, Shandong 250021, P.R. China
}

Received September 5, 2015; Accepted November 4, 2016

DOI: $10.3892 / \mathrm{etm} .2017 .5365$

\begin{abstract}
Rutin has a variety of pharmacological actions, including radical reactivity, and protective activity against lipid peroxidation, viruses and acute pancreatitis; thus, it may be used as a treatment for many diseases. The present study aimed to investigate whether rutin inhibits coronary heart disease through extracellular signal-regulated kinase (ERK) $1 / 2$ and Akt signaling in a porcine model. Male Chinese miniature pigs were randomly divided into four groups: A sham group, a coronary heart disease (CHD) model group, a group receiving $15 \mathrm{mg} / \mathrm{kg}$ rutin for 8 weeks following CHD modeling and a group receiving $45 \mathrm{mg} / \mathrm{kg}$ rutin for 8 weeks following CHD modeling. The results suggested that treatment with rutin suppressed the reduction in left ventricular ejection fraction and increase in systolic internal diameter that occurred in CHD model pigs. Rutin administration reduced the infarct size of the myocardium, attenuated LVEF, increased LVID and inhibited urine protein concentration, BUN and Scr expression levels in CHD model pigs. Results from western blot analysis demonstrated that in CHD pigs treated with $45 \mathrm{mg} / \mathrm{kg}$ rutin, the CHD-associated increases in transforming growth factor $\beta 1$ and SMAD2 expression and reductions in phosphorylated (p)-ERK1/2 and p-Akt expression were attenuated. The present study suggests that rutin inhibits coronary heart disease through ERK1/2 and Akt signaling pathways in a porcine model.
\end{abstract}

\section{Introduction}

In recent years, due to improvements in living standards and an ageing population, the incidence and morbidity

Correspondence to: Dr Guiyue Zhu, Department of Cardiology, Shandong Provincial Hospital Affiliated to Shandong University, 324 Jingwu Road, Jinan, Shandong 250021, P.R. China

E-mail: guiyuezhu@126.com

*Contributed equally

Key words: rutin, coronary heart disease, ERK1/2, Akt of hypertension, coronary heart disease (CHD), diabetes, pulmonary hypertension and other cardiovascular diseases has increased(6 in many countries around the world (1-3). Cardiovascular disease has become one of the major diseases threatening human health. According to the latest statistics from the World Health Organization, each year 17.3 million people succumb to diversified cardiovascular and cerebrovascular diseases, accounting for $\sim 30 \%$ of the global mortality rate, which is considerably higher than the mortality rate of other diseases, including cancer and HIV/AIDS (4). Thus cardiovascular disease has been referred to as the number one enemy of the second health revolution (5).

It has been demonstrated that the transforming growth factor (TGF) $31 /$ SMAD signaling pathway, which participates in the formation and progression of coronary atherosclerosis, remodeling following myocardial infarction, cell apoptosis and inflammatory reactions, is a crucial regulator during the genesis and development of CHD and has become a topic of considerable interest within the field of CHD (6). Different inflammatory cytokines may induce various types of cardiovascular and cerebrovascular diseases in patients with CHD (7). TGF- $\beta 1$ has attracted widespread attention since it serves multiple important roles in certain important disease processes (8). TGF $\beta$ binds a heterodimer of TGF $\beta$ type I and type II receptors (9) and the downstream signaling molecules TGF $\beta 1$, SMAD2 and SMAD3 are phosphorylated and consequently activated (10). Polymers are formed from the activated SMAD2 and SMAD3, together with SMAD4, and are subsequently translocated to the cell nucleus to regulate corresponding gene expression (11).

Rutin, also referred to as rutoside and purple quercetin, is a flavonoid compound that may be obtained from a wide variety of sources (12). Flavonoids, of which rutin is a typical representative, serve a positive role in the treatment of cardiovascular and cerebrovascular diseases (13). Rutin is an active component of medicinally active plants, and a topic of recent research (14). Its effects include maintaining blood vessel elasticity, reducing blood vessel brittleness and lowering capillary permeability, as well as preventing cerebral hemorrhage, retinal hemorrhage and other complications induced by hypertension $(12,15)$. Therefore, the aims of the current study were to investigate whether rutin is able to inhibit CHD and to assess its underlying mechanisms of action on CHD. 


\section{Materials and methods}

Animals and reagents. Rutin was acquired from Sigma-Aldrich (Merck Millipore; Darmstadt, Germany). The chemical structure of rutin is presented in Fig. 1. Male Chinese miniature pigs ( $\mathrm{n}=24$; weight range, $20-30 \mathrm{~kg}$ ) were acquired from the Institute of Laboratory Animal Science, Jining Medical University (Jining, China). Pigs had ad libitum access to a standard laboratory diet (3\% fat) and water and were housed in a climate-controlled farmhouse at $22-24^{\circ} \mathrm{C}$, $60-70 \%$ humidity and a $12-\mathrm{h}$ light-dark cycle. The animals were randomly assigned to one of four groups: CHD model $(\mathrm{n}=6), \mathrm{CHD}$ model $+15 \mathrm{mg} / \mathrm{kg}$ rutin treatment $(\mathrm{n}=6), \mathrm{CHD}$ model $+45 \mathrm{mg} / \mathrm{kg}$ rutin treatment $(\mathrm{n}=6)$, and sham-operated control groups $(n=6)$. Pigs in the CHD model group and the two rutin treatment + CHD groups were fed with a high-fat diet $(20 \%)$ for 2 weeks. The pigs were anesthetized with $30 \mathrm{mg} / \mathrm{kg}$ sodium barbital (Sigma-Aldrich; Merck Millipore) via the ear vein prior to modeling. The common carotid artery was isolated and, following the embedding of a $6 \mathrm{~F}$ arterial sheath and injection of $200 \mathrm{U} / \mathrm{kg}$ heparin, the distal heart was ligated. A guidewire was advanced into the left anterior descending artery and anangioplasty balloon (VistaBrite; Cordis Corporation, Hialeah, FL, USA) was introduced into the left anterior descending branch and pressured with air (10-12 atm) for $30 \mathrm{sec}$ three times, following maintenance of the balloon pressure air gap (1-1.5 atm). Following removal of the catheter and balloon, the common carotid artery was ligated. Pigs in the CHD group were fed a high-fat diet for the following 8 weeks, and those in the CHD model + rutin groups, which were also fed a high-fat diet, were injected with rutin (15 or $45 \mathrm{mg} / \mathrm{kg}$ ) for eight weeks (16). Meanwhile, pigs in the sham group were administered with normal saline. Ethical approval was granted for the present study by the Ethics Committee of the First School of Clinical Medicine at Jining Medical University.

Echocardiography. At the end of the treatment period, anS5-1 linear probe with an iE33 xMatrix Ultrasound instrument (Philips Healthcare, Andover, MA, USA) was used to perform an echocardiographic analysis. The left ventricular ejection fraction (LVEF) and systolic internal diameter (LVID) were calculated.

Blood and urine chemistry. At the end of the treatment period, pigs were anesthetized (20-30 mg/kg pentobarbital sodium; Sigma-Aldrich; Merck Millipore) and blood was sampled through the eyes. Urine protein concentration, blood urea nitrogen (BUN) and plasma creatinine (Scr) levels were measured using ELISA kits (BUN, C013-1; Scr, C011-2; Nanjing Jiancheng, Ltd., Nanjing, China).

Assessment of the infarcted myocardium. All pigs were sacrificed via exsanguination following administration of $30 \mathrm{mg} / \mathrm{kg}$ pentobarbital sodium at 8 weeks after treatment. The left ventricle was washed with physiological saline and refrigerated at $-80^{\circ} \mathrm{C}$ for 5-10 min. Heart tissue samples were cut into $2 \mathrm{~mm}$-thick sections. The tissue sections were stained with 1\% 2,3,5-triphenyltetrazolium chloride (Sigma-Aldrich; Merck Millipore) for $30 \mathrm{~min}$ at room temperature. The infarct

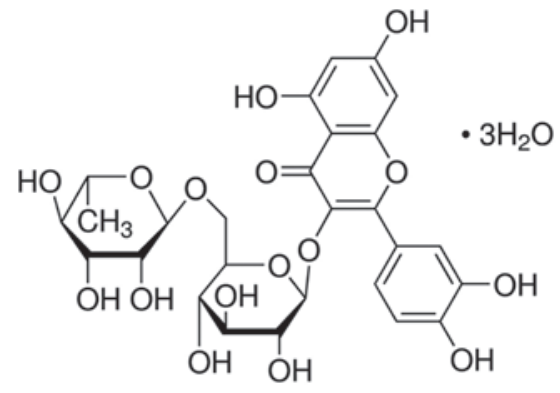

Figure 1. Chemical structure of rutin.

size was measured by volume and weight as a percentage of the left ventricle.

Assessment of antioxidant profile. The left ventricle from each pig was homogenized with radioimmunoprecipitation assay (RIPA) lysis buffer (Nanjing Jiancheng, Ltd.) and centrifuged at $12,000 \mathrm{x} \mathrm{g}$ for $10 \mathrm{~min}$ at $4^{\circ} \mathrm{C}$. Activities of superoxide dismutase (SOD), catalase (CAT), glutathione-S-transferase (GST) and glutathione peroxidase (GSH-Px) were determined using ELISA kits (Wuhan Elabscience Biotechnology Co., Ltd., Wuhan, China) according to the manufacturer's instructions.

Western blot analysis. Heart tissue lysate was prepared using an RIPA lysis buffer (Nanjing Jiancheng, Ltd.) and quantified using the Bradford protein assay (Nanjing Jiancheng Ltd.). Equal amounts of protein $(50 \mu \mathrm{g})$ were separated using 10-12\% dodecyl sulfate-polyacrylamide gel electrophoresis (SDS-PAGE) and electroblotted onto nitrocellulose membranes. Following blocking with $5 \%$ bovine serum albumin (Nanjing Jiancheng, Ltd.) at $37^{\circ} \mathrm{C}$ for $1 \mathrm{~h}$, the membranes were incubated with antibodies against TGF $\beta 1$ (sc-130348; 1:500; Santa Cruz Biotechnology, Inc., Dallas, TX, USA), SMAD2 (sc-101153; 1:500; Santa Cruz Biotechnology, Inc.), phosphorylated-extracellular signal-regulated kinases (p-ERK) 1/2 (sc-136521; 1:500; Santa Cruz Biotechnology, Inc.), anti-p-Akt (sc-7985-R; 1:500; Santa Cruz Biotechnology, Inc.) and $\beta$-actin (sc-47778; 1:2,000; Santa Cruz Biotechnology, Inc.) overnight at $4^{\circ} \mathrm{C}$. Membranes were then incubated with a horseradish peroxidase-conjugated antibody against rabbit or mouse $\mathrm{IgG}$ at a 1:2,000 dilution (sc-2005 and sc-358914; Santa Cruz Biotechnology, Inc.) for $1 \mathrm{~h}$ at room temperature and viewed using enhanced chemiluminescence (ECL) reagent (Nanjing Beyotime Ltd.) in accordance with the manufacturer's instructions. Protein bands were analyzed and quantified using Image J version 3.0 (National Institutes of Health, Bethesda, MD, USA).

Statistical analysis. All values are presented as the mean \pm standard error and were analyzed by analysis of variance and post hoc Bonferroni test. $\mathrm{P}<0.01$ was considered to indicate a statistically significant difference.

\section{Results}

Rutin changes LVEF and LVID values in CHD model pigs. Following treatment with rutin for eight weeks, the mean LVEF of the CHD model pigs was lower than that of the sham 

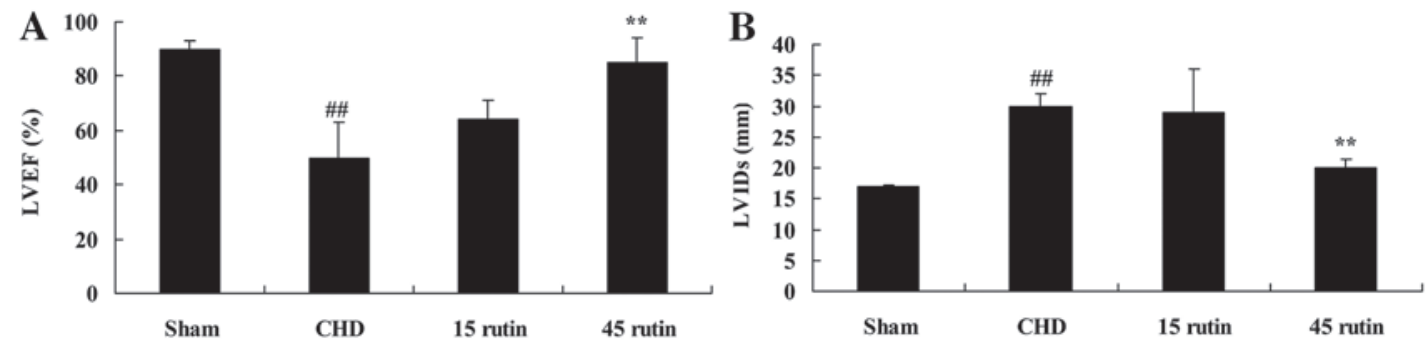

Figure 2. Rutin treatment changes LVEF and LVIDs in CHD model pigs. (A) LVEF percentages and (B) LVIDs in the four groups. $\mathrm{n}=3$ per group. ${ }^{\# \#} \mathrm{P}<0.01$ vs. the sham group; ${ }^{* *} \mathrm{P}<0.01$ vs. the CHD model group. Sham, sham-operated control group; CHD, coronary heart disease model group; $15 \mathrm{rutin}, 15 \mathrm{mg} / \mathrm{kg}$ rutin treated CHD group; 45 rutin, $45 \mathrm{mg} / \mathrm{kg}$ rutin treated CHD group; LVEF, left ventricular ejection fraction; LVID, systolic internal diameter.

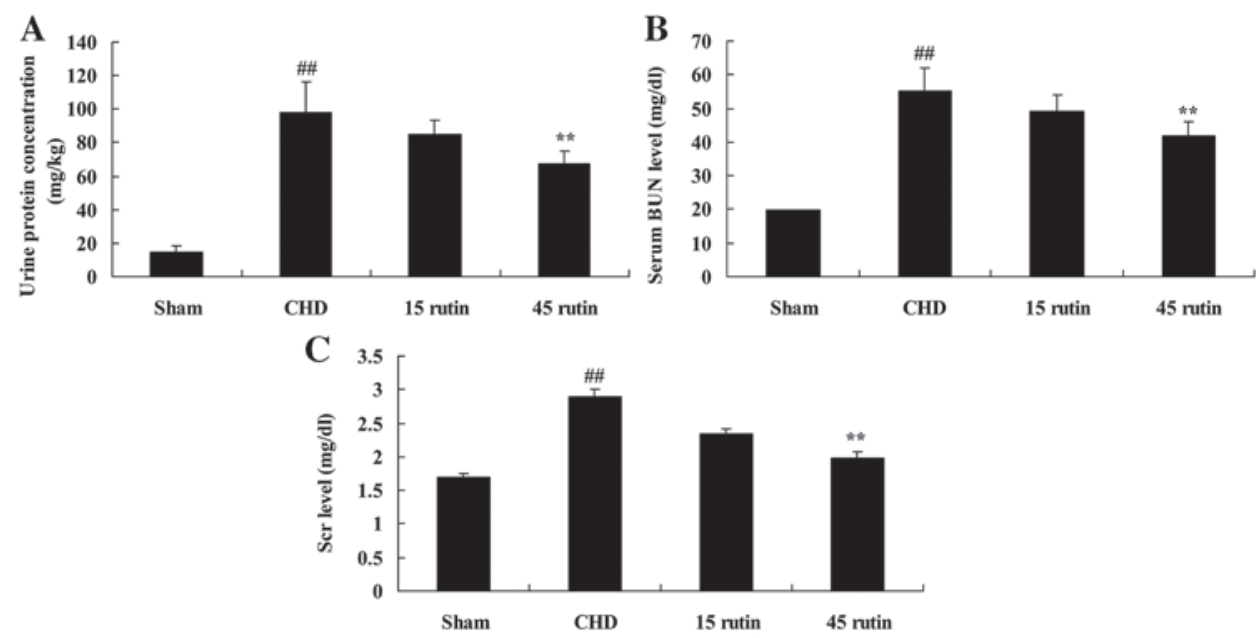

Figure 3. Rutin alters biochemical parameters in CHD model pigs. (A) Protein concentration of urine and expression of (B) BUN and (C) Scr in the four groups. $\mathrm{n}=3$ per group. ${ }^{\# \#} \mathrm{P}<0.01$ vs. the sham group; ${ }^{* *} \mathrm{P}<0.01$ vs. the CHD model group. BUN, blood urea nitrogen; Scr, serum creatinine; sham, sham-operated control group; CHD, coronary heart disease model group; 15 rutin, $15 \mathrm{mg} / \mathrm{kg}$ rutin treated CHD group; 45 rutin, $45 \mathrm{mg} / \mathrm{kg}$ rutin treated CHD group.

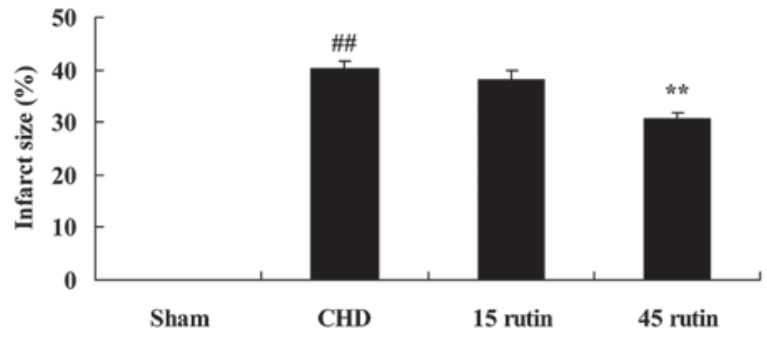

Figure 4. Rutin limits the infarct size of the myocardium in CHD model pigs. $\mathrm{n}=3$ per group. ${ }^{\# \#} \mathrm{P}<0.01$ vs. the sham group; ${ }^{* *} \mathrm{P}<0.01$ vs. the CHD model group. Sham, sham-operated control group; CHD, coronary heart disease model group; 15 rutin, $15 \mathrm{mg} / \mathrm{kg}$ rutin treated CHD group; 45 rutin, $45 \mathrm{mg} / \mathrm{kg}$ rutin treated CHD group.

group $(\mathrm{P}<0.01)$, and the mean LVID of the CHD model pigs was higher than that of the sham group $(\mathrm{P}<0.01)$. However, treatment of CHD model pigs with $45 \mathrm{mg} / \mathrm{kg}$ rutin attenuated LVEF and the increase in LVID that occurred following CHD modeling in the absence of rutin treatment $(\mathrm{P}<0.01$; Fig. 2).

Rutin changes biochemical parameters in CHD model pigs. Compared with the sham-operated control group, the CHD group exhibited a significant elevation of urine protein concentration, serum BUN and Scr expression levels $(\mathrm{P}<0.01$;
Fig. 3). The administration of $45 \mathrm{mg} / \mathrm{kg}$ rutin significantly attenuated the CHD-induced increases in urine protein concentration, BUN and Scr expression levels $(\mathrm{P}<0.01$; Fig. 3).

Rutin reduces infarction of the myocardium in CHD model pigs. Rutin had a protective effect against myocardial infarction in CHD model pigs. CHD induced infarction of the myocardium, which did not occur in the sham-operated control group $(\mathrm{P}<0.01)$. However, the infarct size was significantly reduced following treatment with $45 \mathrm{mg} / \mathrm{kg}$ rutin compared with the infarct size in CHD model pigs ( $\mathrm{P}<0.01$; Fig. 4).

Rutin increases antioxidant activities in CHD model pigs. The CHD model pigs exhibited an inhibited antioxidant profile compared with the sham-operated control group. The activities of the antioxidant enzymes SOD, CAT, GST and GSH-Px were significantly reduced in the CHD group $(\mathrm{P}<0.01$ vs. the sham control; Fig. 5). This reduction in SOD, CAT, GST and GSH-Px activity was significantly attenuated by treatment with $45 \mathrm{mg} / \mathrm{kg}$ rutin $(\mathrm{P}<0.01$; Fig. 5).

Rutin reduces TGF $\beta 1$ expression in CHD model pigs. TGF $\beta 1$ protein expression levels were increased in the CHD model pigs compared with the sham-operated control group $(\mathrm{P}<0.01$; Fig. 6). However, the CHD-induced increase in TGF $\beta 1$ protein 

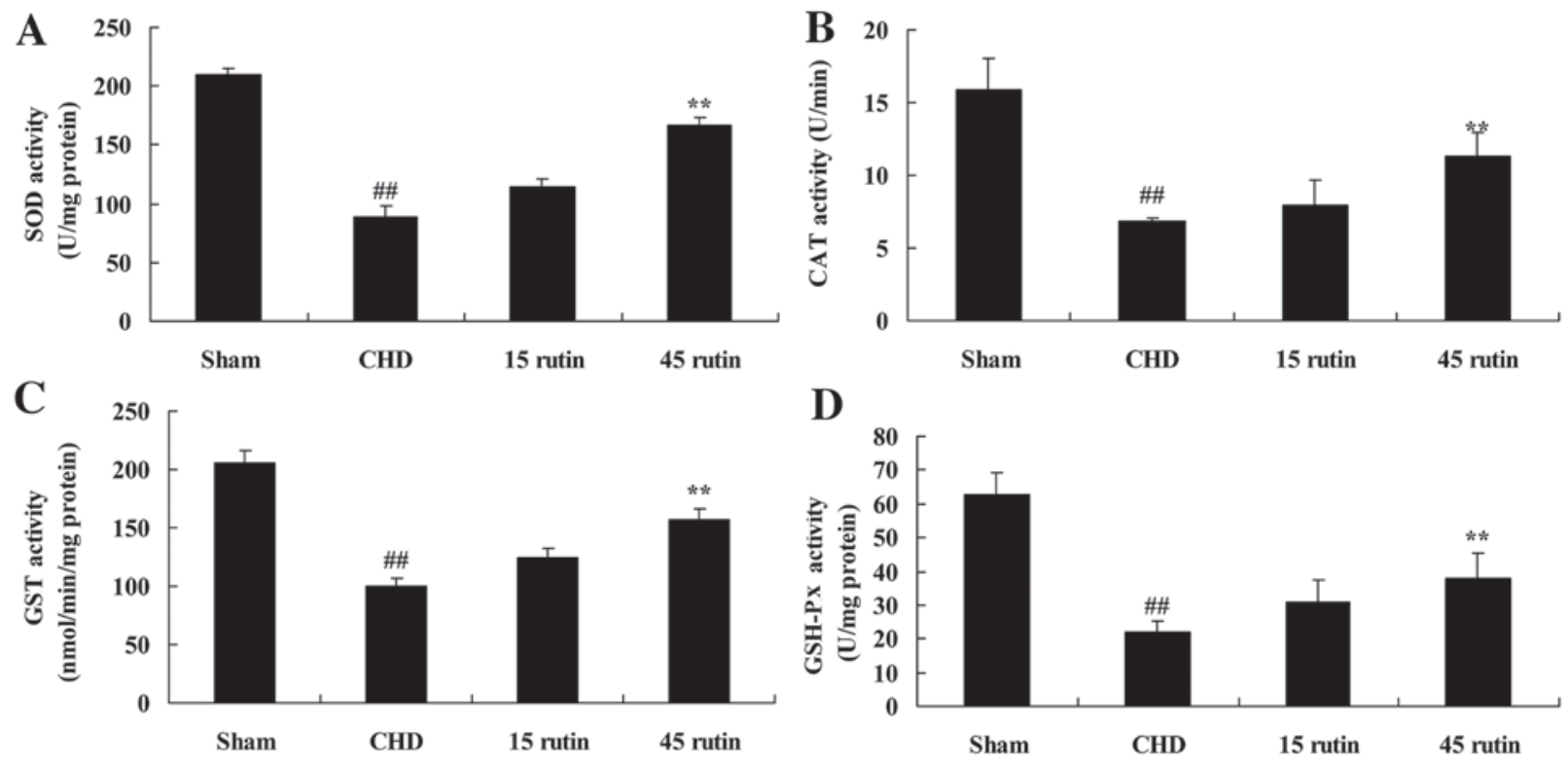

Figure 5. Rutin changes the antioxidant profile in CHD model pigs. (A) SOD, (B) CAT, (C) GST and (D) GSH-Px activity in the four groups. n=3 per group. ${ }^{\# \#} \mathrm{P}<0.01$ vs. the sham group; ${ }^{* *} \mathrm{P}<0.01$ vs. the CHD model group. SOD, superoxide dismutase; CAT, catalase; GST, glutathione-S-transferase; GSH-Px, glutathione peroxidase; sham, sham-operated control group; CHD, coronary heart disease model group; $15 \mathrm{rutin}, 15 \mathrm{mg} / \mathrm{kg}$ rutin treated CHD group; $45 \mathrm{rutin}$, $45 \mathrm{mg} / \mathrm{kg}$ rutin treated CHD group.
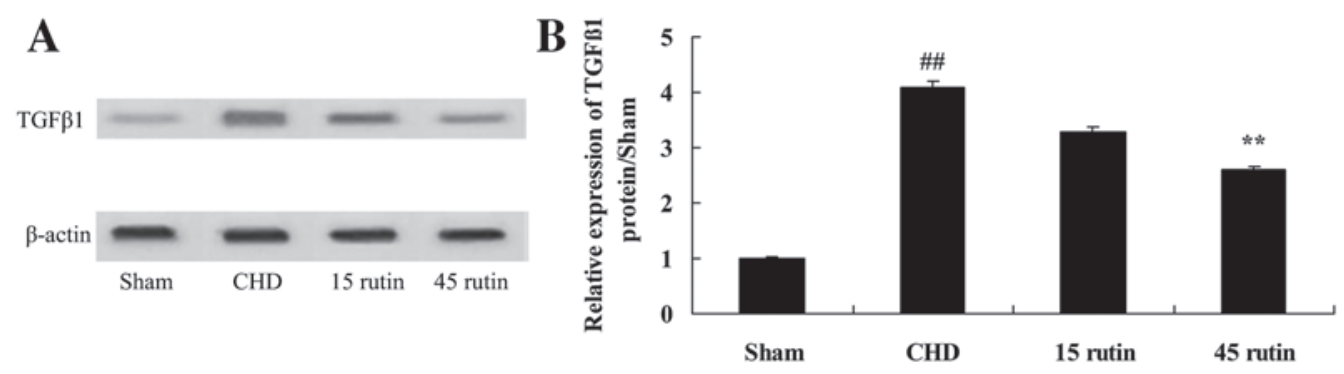

Figure 6. Rutin reduces TGF $\beta 1$ expression in CHD model pigs. (A) Representative western blot and (B) quantitative analysis of TGF $\beta 1$ protein expression in the four groups. $\mathrm{n}=3$ per group. ${ }^{\# \#} \mathrm{P}<0.01$ vs. the sham group; ${ }^{* *} \mathrm{P}<0.01$ vs. the CHD model group. Sham, sham-operated control group; CHD, coronary heart disease model group; 15 rutin, $15 \mathrm{mg} / \mathrm{kg}$ rutin treated CHD group; 45 rutin, $45 \mathrm{mg} / \mathrm{kg}$ rutin treated CHD group; TGF, transforming growth factor.
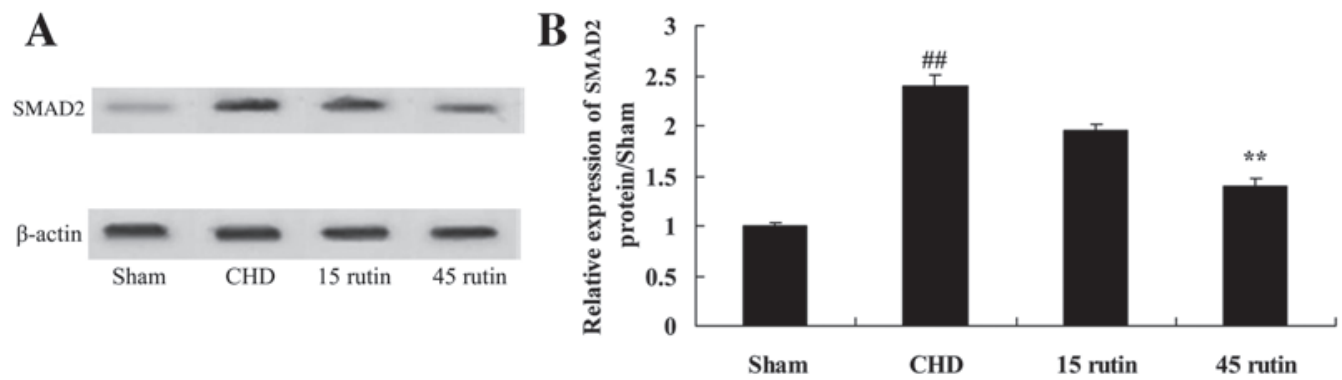

Figure 7. Rutin changes SMAD2 expression in CHD model pigs. (A) Representative western blot and (B) quantitative analysis of SMAD2 protein expression in the four groups. $\mathrm{n}=3$ per group. ${ }^{\# \#} \mathrm{P}<0.01$ vs. the sham group; ${ }^{* *} \mathrm{P}<0.01$ vs. the $\mathrm{CHD}$ model group. Sham, sham-operated control group; CHD, coronary heart disease model group; 15 rutin, $15 \mathrm{mg} / \mathrm{kg}$ rutin treated CHD group; 45 rutin, $45 \mathrm{mg} / \mathrm{kg}$ rutin treated CHD group.

expression was significantly attenuated following treatment with $45 \mathrm{mg} / \mathrm{kg}$ rutin $(\mathrm{P}<0.01$; Fig. 6).

Rutin changes SMAD2 expression in CHD model pigs. Western blotting analyses indicated that expression of SMAD2 protein was increased in the CHD pigs compared with the sham-operated control group $(\mathrm{P}<0.01 ;$ Fig. 7). However, the
CHD model pigs that received treatment with $45 \mathrm{mg} / \mathrm{kg}$ rutin exhibited decreased expression levels of SMAD2 protein compared with the CHD group $(\mathrm{P}<0.01)$.

Rutin increases p-ERK1/2 expression in CHD model pigs. There was a reduction in $\mathrm{p}$-ERK1/2 protein expression in the CHD model pigs compared with the sham-operated control 
A

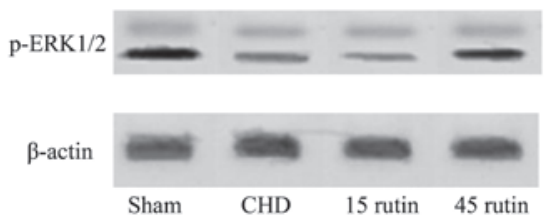

B

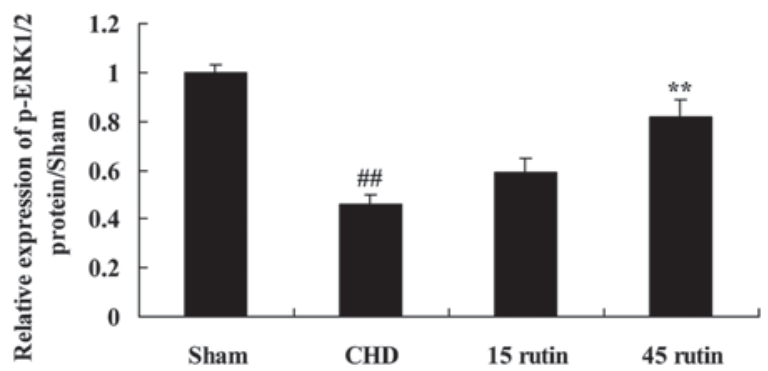

Figure 8. Rutin changes p-ERK1/2 expression in CHD model pigs. (A) Representative western blot and (B) quantitative analysis of p-ERK1/2 protein expression in the four groups. $\mathrm{n}=3$ per group. ${ }^{\# \#} \mathrm{P}<0.01$ vs. the sham group; ${ }^{* *} \mathrm{P}<0.01$ vs. the CHD model group. p-ERK1/2, phosphorylated extracellular signal-regulated kinase; sham, sham-operated control group; CHD, coronary heart disease model group; 15 rutin, 15 mg/kg rutin treated CHD group; 45 rutin, 45 mg/kg rutin treated CHD group.
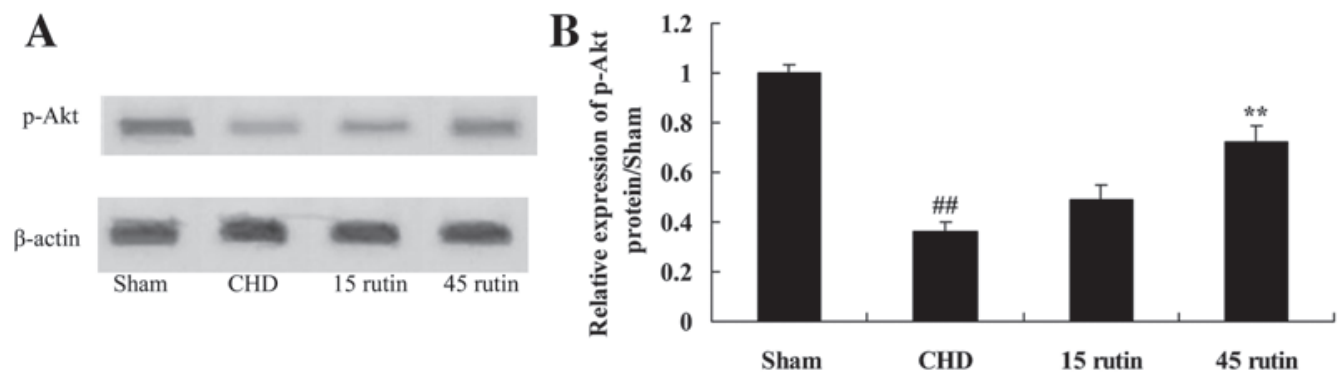

Figure 9. Rutin changes p-Akt expression in CHD model pigs. (A) Representative western blot and (B) quantitative analysis of p-Akt protein expression in CHD model pigs. $\mathrm{n}=3$ per group. ${ }^{\# \#} \mathrm{P}<0.01$ vs. the sham group; ${ }^{* *} \mathrm{P}<0.01$ vs. the CHD model group. Sham, sham-operated control group; $\mathrm{CHD}$, coronary heart disease model group; 15 rutin, $15 \mathrm{mg} / \mathrm{kg}$ rutin treated CHD group; 45 rutin, $45 \mathrm{mg} / \mathrm{kg}$ rutin treated CHD group.

group (P<0.01; Fig. 8). However, p-ERK1/2 protein expression was restored in CHD model pigs treated with $45 \mathrm{mg} / \mathrm{kg}$ rutin compared with the untreated CHD group $(\mathrm{P}<0.01$; Fig. 8).

Rutin increases p-Akt expression in CHD model pigs. Compared with the sham-operated control group, p-Akt protein expression of the CHD model pigs was suppressed $(\mathrm{P}<0.01$; Fig. 9). Treatment with rutin increased the $\mathrm{p}-\mathrm{Akt}$ protein expression in the CHD model pigs $(\mathrm{P}<0.01$; Fig. 9).

\section{Discussion}

CHD is a set of clinical syndromes presenting as different degrees of myocardial ischemia caused by coronary atherosclerotic plaque rupture, which is accompanied by platelet aggregation, thrombosis and different degrees of stenosis that may involve the complete occlusion of coronary vessels (17). It poses a great threat to human health and life due to its characteristics of emergent onset, further complications and a high mortality rate $(18,19)$. The morbidity and mortality of coronary heart disease in China has been gradually rising, and the rate of increase appears to be escalating $(20,21)$. In the present study of a porcine model of CHD, rutin treatment significantly increased the LVEF, reduced the LVID, attenuated the increase in urine protein concentration, BUN and Scr expression levels induced by CHD, and limited the area of infarcted myocardium.

Oxidative stress occurs when the body is exposed to various types of noxious stimuli, and excessive quantities of highly reactive molecules, such as reactive oxygen species and reactive nitrogen species are generated (20). When the generation of oxidative species exceeds their removal, an imbalance in the oxidation and antioxidant system occurs, leading to vascular endothelial tissue damage (21). The oxidized low-density lipoprotein cholesterol (OX-LDL) that is generated by oxidative stress has an important effect on atherosclerosis (22). It directly and indirectly contributes to atherosclerosis via a variety of mechanisms, including endothelial cell injury and endothelium dysfunction; in addition, LDL in the circulation is promoted to enter subcutaneous clearance (23). LDL has a chemotactic effect on monocytes and T lymphocytes (24). Furthermore, it may inhibit the activity of macrophages, causing them to remain within the arterial wall to increase inflammatory reactions of the local blood vessel wall (25). The present study demonstrated that treatment with rutin significantly reversed the $\mathrm{CHD}$-induced reductions of SOD, CAT, GST and GSH-Px activities in CHD model pigs. This builds on the results from Yu et al (14) who previously found that rutin inhibits amylin-induced neurocytotoxicity and oxidative stress.

The signaling pathways associated with TGF $\beta 1 /$ SMAD2 serve an important role in the biological functions of TGF $\beta 1$, including induction of cell proliferation, immune suppression and inflammation (26). A significant enhancement was previously observed between TGF $\beta 1$ expression in patients with hypertrophic cardiomyopathy and dilated cardiomyopathy in the animal model (27). In addition, a number of studies have indicated that the TGF $\beta$ signaling pathway may inhibit the inflammatory gene expression caused by myocardial infarction with inflammatory infiltration $(28,29)$. TGF $\beta 1$ is of great significance in various pathological processes, including infarction, myocardial remodeling and interstitial fibrosis (30). 
An analysis of TGF $\beta 1$ and SMAD2 levels and their correlation with CHD risk factors indicated that TGF $\beta 1$ is associated with the expression of lipoprotein A and uric acid $(31,32)$. Increased levels of lipoprotein $\mathrm{A}$ and uric acid are considered as markers of atherosclerosis, which indicates that TGF $\beta 1$ may regulate atherosclerosis, thereby accelerating the establishment of CHD. The level of SMAD2 is closely associated with blood glucose content, which indicates that SMAD2 may affect the onset and development of CHD and diabetes mellitus (11). The current study observed that treatment with rutin significantly suppressed the CHD-induced TGF $\beta 1 /$ SMAD2 signaling pathway in CHD model pigs. This is in accordance with a previous study by Han et al (16), which suggested that rutin ameliorates renal fibrosis and proteinuria by activating TGF $\beta 1$-SMAD2 signaling in 5/6-nephrectomized rats.

The ERK1/2 signaling pathway is a typical mitogen-activated protein kinase (MAPK) signal transduction pathway (33). It may be activated by insulin, growth factors and serum (33). Furthermore, it is activated through the phosphorylation of numerous types of growth factors, ion beam radiation and hydrogen peroxide (34). ERK1/2 enters the cell nucleus and acts as a transcription factor, accelerating the transcription and expression of certain genes and is closely associated with cell proliferation and differentiation (35). Studies have indicated that ERK1/2 mediates the stress and inflammatory response $(36,37)$. ERK1/2 is an essential signal transduction pathway in the MAPK family and it has been demonstrated that ERK1/2 is closely associated with atherosclerosis (38). Jeong et al (39) suggested that rutin inhibits myocardial ischemia/reperfusion-induced apoptosis via ERK1/2 and PI3 K/Akt signaling in vitro, and the current study indicated that treatment with rutin recovered $\mathrm{p}-\mathrm{ERK} 1 / 2$ protein expression in $\mathrm{CHD}$ model pigs.

Akt activates NF- $\kappa \mathrm{B}$ and other nuclear transcription factors, produces a large number of pro-inflammatory cytokines and induces or maintains inflammation (40). It also stimulates sustained cell growth, inhibits cell apoptosis and promotes carcinogenesis (40). In the present study, it was demonstrated that treatment with rutin increased p-Akt protein expression levels in CHD model pigs. This is in agreement with a study by Moghbelinejad et al (41), which indicated that rutin may be protective against the neurotoxic effects of amyloid $\beta$ on memory in rats through actions on ERK and PI3K/Akt.

In conclusion, rutin inhibits CHD in a porcine model; this may be due to an anti-oxidative effect, suppression of TGF $\beta 1$ and SMAD2 and the modulation of ERK1/2 and Akt signaling. The results of the current study support the notion that rutin may be developed as a novel therapeutic strategy to treat CHD in the future.

\section{Acknowledgements}

The present study was supported by a grant from the Natural Science Foundation of Shandong Province (grant no. Q2007C10).

\section{References}

1. Ueno T, Chow LW and Toi M: Increases in circulating VEGF levels during COX-2 inhibitor treatment in breast cancer patients. Biomed Pharmacother 60: 277-279, 2006.
2. Chuah BY, Putti T, Salto-Tellez M, Charlton A, Iau P, Buhari SA, Wong CI, Tan SH, Wong AL, Chan CW, et al: Serial changes in the expression of breast cancer-related proteins in response to neoadjuvant chemotherapy. Ann Oncol 22: 1748-1754, 2011

3. Kullo IJ, Jouni H, Olson JE, Montori VM and Bailey KR: Design of a randomized controlled trial of disclosing genomic risk of coronary heart disease: The myocardial infarction genes (MI-GENES) study. BMC Med Genomics 8: 51, 2015.

4. Pradeepa R, Surendar J, Indulekha K, Chella S, Anjana RM and Mohan V: Prevalence of metabolic syndrome and its association with coronary artery disease among an urban elderly south indian population (CURES- 145). J Assoc Physicians India 64: 20-25, 2016.

5. Nissen SE, Yeomans ND, Solomon DH, Lüscher TF, Libby P, Husni ME, Graham DY, Borer JS, Wisniewski LM, Wolski KE, et al: Cardiovascular safety of celecoxib, naproxen, or ibuprofen for arthritis. N Engl J Med 375: 2519-2529, 2016.

6. Shirai T, Nazarewicz RR, Wallis BB, Yanes RE, Watanabe R, Hilhorst M, Tian L, Harrison DG, Giacomini JC, Assimes TL, et al: The glycolytic enzyme PKM2 bridges metabolic and inflammatory dysfunction in coronary artery disease. J Exp Med 213: 337-354, 2016.

7. Li H, Sun K, Zhao R, Hu J, Hao Z, Wang F, Lu Y, Liu F and Zhang Y: Inflammatory biomarkers of coronary heart disease. Front Biosci (Landmark Ed) 22: 504-515, 2017.

8. Li S, Fan Q, He S, Tang T, Liao Y and Xie J: MicroRNA-21 negatively regulates treg cells through a TGF- $\beta 1 /$ Smad-independent pathway in patients with coronary heart disease. Cell Physiol Biochem 37: 866-878, 2015.

9. Thakur A, Schalk D, Tomaszewski E, Kondadasula SV, Yano H, Sarkar FH and Lum LG: Microenvironment generated during EGFR targeted killing of pancreatic tumor cells by ATC inhibits myeloid-derived suppressor cells through COX2 and PGE2 dependent pathway. J Transl Med 11: 35, 2013.

10. Schaan BD, Quadros AS, Sarmento-Leite R, De Lucca G Jr, Bender A and Bertoluci M: 'Correction:' Serum transforming growth factor beta-1 (TGF-beta-1) levels in diabetic patients are not associated with pre-existent coronary artery disease. Cardiovasc Diabetol 6: 19, 2007.

11. Yang J, Zeini M, Lin CY, Lin CJ, Xiong Y, Shang C, Han P, Li W, Quertermous T, Zhou B and Chang CP: Epicardial calcineurin-NFAT signals through Smad2 to direct coronary smooth muscle cell and arterial wall development. Cardiovasc Res 101: 120-129, 2014.

12. Hosseinzadeh $\mathrm{H}$ and Nassiri-Asl M: Review of the protective effects of rutin on the metabolic function as an important dietary flavonoid. J Endocrinol Invest 37: 783-788, 2014.

13. Chuffa LG, Fioruci-Fontanelli BA, Bordon JG, Pires RB, Braga CP, Seiva FR and Fernandes AA: Rutin ameliorates glycemic index, lipid profile and enzymatic activities in serum, heart and liver tissues of rats fed with a combination of hypercaloric diet and chronic ethanol consumption. Indian J Biochem Biophys 51: 215-222, 2014.

14. Yu XL, Li YN, Zhang H, Su YJ, Zhou WW, Zhang ZP, Wang SW, Xu PX, Wang YJ and Liu RT: Rutin inhibits amylin-induced neurocytotoxicity and oxidative stress. Food Funct 6: 3296-3306, 2015.

15. Benavente-Garcia O and Castillo J: Update on uses and properties of citrus flavonoids: New findings in anticancer, cardiovascular and anti-inflammatory activity. J Agric Food Chem 56: 6185-6205, 2008.

16. Han Y, Lu JS, Xu Y, Zhang L and Hong BF: Rutin ameliorates renal fibrosis and proteinuria in 5/6-nephrectomized rats by anti-oxidation and inhibiting activation of TGF $\beta 1$-smad signaling. Int J Clin Exp Pathol 8: 4725-4734, 2015.

17. Ammenwerth E, Woess S, Baumgartner C, Fetz B, van der Heidt A, Kastner P, Modre-Osprian R, Welte S and Poelzl G: Evaluation of an integrated telemonitoring surveillance system in patients with coronary heart disease. Methods Inf Med 54: 388-397, 2015.

18. Salfati E, Nandkeolyar S, Fortmann SP, Sidney S, Hlatky MA, Quertermous T, Go AS, Iribarren C, Herrington DM, Goldstein BA and Assimes TL: Susceptibility loci for clinical coronary artery disease and subclinical coronary atherosclerosis throughout the life-course. Circ Cardiovasc Genet 8: 803-811, 2015.

19. Zhang H, Jiang Y, Nguyen HD, Poo DC and Wang W: The effect of a smartphone-based coronary heart disease prevention (SBCHDP) programme on awareness and knowledge of $\mathrm{CHD}$, stress and cardiac-related lifestyle behaviours among the working population in Singapore: A pilot randomised controlled trial. Health Qual Life Outcomes 15: 49, 2017. 
20. Riegersperger M, Covic A and Goldsmith D: Allopurinol, uric acid and oxidative stress in cardiorenal disease. Int Urol Nephrol 43: 441-449, 2011.

21. Prasad K and Dhar I: Oxidative stress as a mechanism of added sugar-induced cardiovascular disease. Int J Angiol 23: 217-226, 2014.

22. Turan T, Menteşe Ü, Ağaç MT, Akyüz AR, Kul S, Aykan AÇ, Bektas H, Korkmaz L, Öztas Menteșe S, Dursun İ and Celik S: The relation between intensity and complexity of coronary artery lesion and oxidative stress in patients with acute coronary syndrome. Anatol J Cardiol 15: 795-800, 2015.

23. Zhang J, Wang M, Li Z, Bi X, Song J, Weng S and Fu G: NADPH oxidase activation played a critical role in the oxidative stress process in stable coronary artery disease. Am J Transl Res 8: 5199-5210, 2016.

24. Zurgil N, Solodeev I, Gilburd B, Shafran Y, Afrimzon E, Avtalion R, Shoenfeld Y and Deutsch M: Monitoring the apoptotic process induced by oxidized low-density lipoprotein in Jurkat T-lymphoblast and U937 monocytic human cell lines. Cell Biochem Biophys 40: 97-113, 2004.

25. Pintér Ö, Hardi P, Nagy T, Gasz B, Kovács V, Arató E, Sínay L, Lénárd L and Jancsó G: The role of GST polymorphism in reperfusion induced oxidative stress, inflammatory responses and clinical complications after surgical and percutaneous coronary intervention. Clin Hemorheol Microcirc 66: 261-272, 2017.

26. Crobu F, Palumbo L, Franco E, Bergerone S, Carturan S, Guarrera S, Frea S, Trevi G, Piazza A and Matullo G: Role of TGF-beta1 haplotypes in the occurrence of myocardial infarction in young Italian patients. BMC Med Genet 9: 13, 2008.

27. Purnomo Y, Piccart Y, Coenen T, Prihadi JS and Lijnen PJ: Oxidative stress and transforming growth factor-betal-induced cardiac fibrosis. Cardiovasc Hematol Disord Drug Targets 13: 165-172, 2013

28. Guo L, Zhang Y,Zhang L, Huang F, Li J and Wang S: MicroRNAs, TGF- $\beta$ signaling and the inflammatory microenvironment in cancer. Tumour Biol 37: 115-125, 2016.

29. Shen $Y$, Zhang $C$ and Chen Y: TGF- $\beta$ in inflammatory bowel diseases: A tale of the janus-like cytokine. Crit Rev Eukaryot Gene Expr 25: 335-347, 2015.

30. Valls N, Gormaz JG, Aguayo R, González J, Brito R, Hasson D, Libuy M, Ramos C, Carrasco R, Prieto JC, et al: Amelioration of persistent left ventricular function impairment through increased plasma ascorbate levels following myocardial infarction. Redox Rep 21: 75-83, 2016

31. Forbes K, Souquet B, Garside R, Aplin JD and Westwood M: Transforming growth factor-\{beta\} (TGF $\{$ beta\}) receptors I/II differentially regulate TGF $\{$ beta 1 and IGF-binding protein-3 mitogenic effects in the human placenta. Endocrinology 151: $1723-1731,2010$
32. Thu VT, Kim HK, Long le T, Thuy TT, Huy NQ, Kim SH, Kim N, Ko KS, Rhee BD and Han J: NecroX-5 exerts anti-inflammatory and anti-fibrotic effects via modulation of the TNF $\alpha / D c n / T G F \beta 1 / S m a d 2$ pathway in hypoxia/reoxygenation-treated rat hearts. Korean J Physiol Pharmacol 20: 305-314, 2016.

33. Zhong Y, Cheng CF, Luo YZ, Tian CW, Yang H, Liu BR, Chen MS, Chen YF and Liu SM: C-reactive protein stimulates RAGE expression in human coronary artery endothelial cells in vitro via ROS generation and ERK/NF- $\kappa B$ activation. Acta Pharmacol Sin 36: 440-447, 2015.

34. Tarone G, Sbroggiò M and Brancaccio M: Key role of ERK1/2 molecular scaffolds in heart pathology. Cell Mol Life Sci 70: 4047-4054, 2013.

35. Yu J, Wang L, Akinyi M, Li Y, Duan Z, Zhu Y and Fan G: Danshensu protects isolated heart against ischemia reperfusion injury through activation of Akt/ERK1/2/Nrf2 signaling. Int J Clin Exp Med 8: 14793-14804, 2015.

36. Lee YH, Lee SJ, Jung JE, Kim JS, Lee NH and Yi HK: Terrein reduces age-related inflammation induced by oxidative stress through Nrf2/ERK1/2/HO-1 signalling in aged HDF cells. Cell Biochem Funct 33: 479-486, 2015.

37. Hao Q, Chen X, Wang X, Dong B and Yang C: Curcumin attenuates angiotensin ii-induced abdominal aortic aneurysm by inhibition of inflammatory response and ERK signaling pathways. Evid Based Complement Alternat Med 2014: 270930, 2014.

38. Pang X, Liu J, Li Y, Zhao J and Zhang X: Emodin inhibits homocysteine-induced c-reactive protein generation in vascular smooth muscle cells by regulating ppargamma expression and ROS-ERK1/2/p38 signal pathway. PLoS One 10: e0131295, 2015.

39. Jeong JJ, Ha YM, Jin YC, Lee EJ, Kim JS, Kim HJ, Seo HG, Lee JH, Kang SS, Kim YS and Chang KC: Rutin from Lonicera japonica inhibits myocardial ischemia/reperfusion-induced apoptosis in vivo and protects $\mathrm{H} 9 \mathrm{c} 2$ cells against hydrogen peroxide-mediated injury via ERK1/2 and PI3K/Akt signals in vitro. Food Chem Toxicol 47: 1569-1576, 2009.

40. Hamamdzic D, Fenning RS, Patel D, Mohler ER III, Orlova KA, Wright AC, Llano R, Keane MG, Shannon RP, Birnbaum MJ and Wilensky RL: Akt pathway is hypoactivated by synergistic actions of diabetes mellitus and hypercholesterolemia resulting in advanced coronary artery disease. Am J Physiol Heart Circ Physiol 299: H699-H706, 2010.

41. Moghbelinejad S, Nassiri-Asl M, Farivar TN, Abbasi E, Sheikhi M, Taghiloo M, Farsad F, Samimi A and Hajiali F: Rutin activates the MAPK pathway and BDNF gene expression on beta-amyloid induced neurotoxicity in rats. Toxicol Lett 224: 108-113, 2014. 